\section{HAUTKREBSSCREENING}

\section{Nicht bei allen Hautärzten eine Kassenleistung}

— Neun von zehn niedergelassenen Dermatologen beteiligen sich an der Hautkrebsfrüherkennung. Im Schnitt screent jeder Hautarzt 345 Patienten im Quartal. Doch die Einführung der Hautkrebsfrüherkennung als GKV-Leistung wird von den Dermatologen unterschiedlich bewertet. Neben Befürwortern sehen Viele in dem Screening auch weiterhin eher eine IGeLeistung, wie Prof. Dr. M. Augustin aus Hamburg berichtete. Entsprechend geben die Befragten auch unterschiedliche Ab- rechnungsmodalitäten an: 38\% der Dermatologen bieten die Untersuchung als Kassenleistung an, $8 \%$ nur als IGeL, viele als Mischform.

Die Gesamtbeurteilung des HautkrebsScreenings als Kassenleistung fällt dennoch positiv aus: Die Hautärzte glauben überwiegend, dass dadurch die Versorgung von Patienten mit Hauttumoren besser geworden ist. Den befragten Dermatologen zufolge wird übrigens jeder fünfte Patient vom Hausarzt zugewiesen.

\title{
SCHWERER FALL VON CYBERCHONDRIE
}

\section{Kennen Sie Morgellons Krankheit?}

- Morgellons Krankheit hat Deutschland erreicht. Die zunächst von US-amerikanischen Selbsthilfegruppen kolportierte Erkrankung zeichnet sich angeblich durch Fasern aus, die aus Geschwüren der Haut hervorbrechen. Als Ursache vermuten die Betroffenen unter anderem gentechnisch veränderte Lebensmittel oder unbekannte Mikroorganismen. Durch Internetforen haben sich diese Wahninhalte weltweit verbreitet.

PD Dr. Wolfgang Harth, Vorsitzender des Arbeitskreises Psychosomatische Dermatologie aus Berlin, berichtete jetzt von einem Fall einer solchen „Cyberchondrie“. Eine 53-Jährige litt an nicht heilenden Ulzerationen im Gesicht und am Hals. Ein Jahr lang wendete sie ununterbrochen
Antimykotika und Antibiotika aus unterschiedlichen Quellen an. Nach 18 Arztkonsultationen wurde sie schließlich stationär aufgenommen. Sie hatte die Ursache ihrer Hautulzera, die sich als textile Fasern herausstellten, fein säuberlich gesammelt. Zudem hatte sie detaillierte Zeichnungen zur Lokalisation der Erkrankung inklusive Hautquerschnitten erstellt. Einen Zusammenhang der Läsionen mit den zur Hautmanipulation verwendeten Pinzetten leugnete sie.

Stationär sprach die Patientin auf eine antiseptische Lokaltherapie und eine auf die Symptomatik abgestimmte Medikation mit Neuroleptika an. In der ambulanten Situation setzte sie die Medikation aber ab und erlitt sofort ein Rezidiv.

\section{ALLERGISCHE KREUZREAKTION}

\section{Anaphylaxie nach Neuraminidasehemmer-Einnahme}

- Solange keine Impfstoffe bereitstehen, ruht bei neu aufgetretenen Influenzastämmen wie der Schweinegrippe die ganze Hoffnung auf Virostatika wie Oseltamivir. Allerdings sind selten anaphylaktische Reaktionen möglich.

Prof. Dr. Hans Merk aus Aachen berichtete über einen Fall einer Anaphylaxie nach Einnahme des Grippemittels auf dem Boden eines Beifuß-Gewürzmittel-Syndroms. Ne- ben einer Beifußsensibilisierung waren in diesem Fall auch Kreuzsensibilisierungen auf Möhre, Sellerie und Gewürze, unter anderem auch Sternanis nachzuweisen. Der chinesische Sternanis liefert den Grundstoff zur Herstellung von Oseltamivir, die Shikimisäure. Das Risiko dürfte aber begrenzt sein, denn diese Basissubstanz wird heute überwiegend aus gentechnisch veränderten Stämmen von Escherichia coli gewonnen.

\section{STUDIE ZUR PSORIASISTHERAPIE}

\section{Zweiklassenmedizin existiert nicht}

— Gesetzlich Krankenversicherte mit mittelschwerer bis schwerer Psoriasis erhalten nicht seltener eine systemische oder eine Biologikatherapie als Privatversicherte. Das zeigte eine Untersuchung des Instituts für Demoskopie in Allensbach. Im Gegenteil, systemische Therapeutika wurden sogar etwas häufiger verordnet, was aber der etwas größeren Krankheitsschwere im Kollektiv der GKV-Versicherten entsprach. Trotz der gleich guten Versorgung waren die GKV-Versicherten insgesamt aber unzufriedener mit ihrer Gesundheitsversorgung und ihrer Krankenversicherung. Zwischen den GKVen gab es aber große Unterschiede: Die Zufriedenheit der Versicherten der Techniker Krankenkasse war ebenso hoch wie bei Versicherten von Privatkassen.

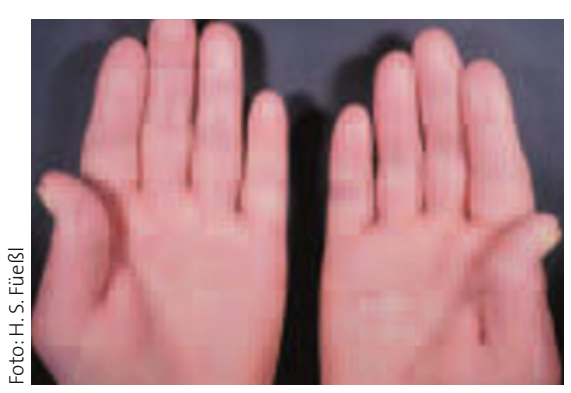

GKV-Patienten erhalten nicht seltener als Privatversicherte teure Psoriasistherapien.

\section{NICHT AUF ANAMNESE VERLASSEN}

\section{Kontrastmittelallergie jederzeit möglich}

— Die Anamnese bezüglich einer Reaktion auf Kontrastmittel ist wenig aussagekräftig für die Prognose einer weiteren Reaktion. Wie Prof. Dr. Merk, Aachen, berichtete, erleiden genauso viele Patienten ohne Hinweise in der Anamnese eine solche Reaktion wie Patienten mit einer vorangegangenen Kontrastmittelreaktion. Möglicherweise spielt hier eine direkte Interaktion der Kontrastmittel mit den T-Lymphozyten eine Rolle.

FRIEDERIKE KLEIN -

- 45. Kongress der Deutschen Gesellschaft für Dermatologie, Dresden 29.4.-2.5.2009 